\title{
4th EPMA/IFCARS Workshop on Personalized Medicine and ICT
}

Chairs: Olga Golubnitschaja, MD (D), Heinz U. Lemke, PhD (D) 